Annuaire suisse de politique de développement

22-2 | 2003

Société de l'information et coopération internationale

\title{
Les technologies de l'information au Burkina Faso : une course de fond
}

Sylvestre Ouédraogo

\section{(2) OpenEdition}

Édition électronique

URL : http://journals.openedition.org/aspd/547

DOI : 10.4000/aspd.547

ISSN : 1663-9669

Éditeur

Institut de hautes études internationales et du développement

Édition imprimée

Date de publication : 1 novembre 2003

Pagination : 77-79

ISSN : 1660-5934

Référence électronique

Sylvestre Ouédraogo, "Les technologies de l'information au Burkina Faso : une course de fond",

Annuaire suisse de politique de développement [En ligne], 22-2 | 2003, mis en ligne le 22 mars 2010

consulté le 08 septembre 2020. URL : http://journals.openedition.org/aspd/547 ; DOI : https://doi.org/ $10.4000 / a s p d .547$ 


\title{
Les technologies de l'information au Burkina Faso: une course de fond
}

\author{
Sylvestre Ouédraogo*
}

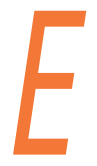

veille ton intelligence et participe au progrès du monde... La devise de l'association Yam Pukri - qui a pour objectif de vulgariser l'informatique et l'Internet au Burkina Faso - sous-entend que les TIC ne sont pas un luxe pour l'Afrique, mais un droit à participer au monde. Pionnière dans ce secteur, l'association Yam Pukri, fondée en 1998, a formé plus de 3000 personnes et créé six centres d'accès collectifs à Internet.

Le Burkina Faso est du «mauvais côté» de la fracture numérique. La situation est elle-même très déséquilibrée entre la capitale, les villes secondaires et les zones rurales. En 2001, le Burkina Faso avait une télédensité d'environ 1 pour 100 habitants. Avec 12 millions d'habitants, le pays compte seulement 58'000 lignes téléphoniques principales, soit l'équivalent d'une petite ville européenne. En outre, en novembre 2002, on comptait 4100 accès collectifs au téléphone (télécentres). En forte progression $-51 \%$ en moins de deux ans -, ce parc comporte néanmoins de grosses disparités, puisque $75 \%$ des télécentres sont à Ouagadougou, la capitale, et $7 \%$ à Bobo-Dioulasso, la deuxième ville du pays. En ce qui concerne les accès collectifs à Internet, ils sont estimés à $150^{1}$ pour la ville de Ouagadougou.

La stratégie de Yam Pukri est de toucher les publics les plus intéressés par les nouvelles technologies pour ensuite créer un effet d'entraînement. Les centres de formation de l'association sont situés dans les milieux scolaires ou urbains. Parmi les six centres, deux sont installés dans des lycées et collèges, trois dans des espaces publics et un dans une organisation de développement. Quatre sont à Ouagadougou et deux dans des villes de province, Bobo-Dioulasso et Banfora.

Toutes les personnes bénéficiant des services de Yam Pukri en sont membres. Les animateurs et les responsables de commissions - animation de sites web, maintenance informatique, forums d'échanges Internet, étude et observation des TIC au Burkina Faso, analyse des données numériques - composent l'équipe active des membres. La plus grande tâche des animateurs consiste à identifier les bénéficiaires des formations, et à concevoir des types de formation

* Enseignant en économie, président-fondateur de Yam Pukri, Burkina Faso.

Ces informations proviennent de la DELGI (Délégation générale à l'informatique) du Burkina et datent de fin 2001. On peut estimer que le nombre de cybercafés atteint aujourd'hui les 250 si l'on compte les télécentres connectés (un à trois ordinateurs). 
adaptés aux niveaux scolaires et aux besoins spécifiques de chacun. Cette approche personnalisée a amené à développer une dizaine de modules, chacun accompagné d'une évaluation et d'une attestation de formation. Certains modules sont destinés à des recherches d'emplois ciblés (aide secrétaire, opérateur de saisie, aidecomptable, animateur de cybercafé, assistant en maintenance informatique ou encore assistant de travaux multimédias). D'autres formations permettent aux étudiants de se former aux TIC, mais aussi de faire des stages et de mener des travaux en rapport avec les nouvelles technologies (mémoires, recherches, etc.) avec l'aide de l'association.

L'apprentissage des TIC passe aussi par une compréhension propre du phénomène pour définir ce qui est adéquat dans son contexte. Ainsi, l'association mène des études et des enquêtes sur l'évolution et les usages des TIC au Burkina Faso. Yam Pukri est maintenant sollicitée aux niveaux national et international pour partager ses analyses.

Les besoins en formation et en accès sont très différents d'un contexte à l'autre. La plupart des utilisateurs burkinabés ne se servent pas d'un ordinateur quotidiennement, et le volume de transaction est très réduit. La possession d'un ordinateur et d'un accès personnalisé est donc rarement nécessaire. La collectivisation des outils est une source d'économie et d'efficacité. L'accès collectif permet de réduire les coûts de formation de matériel, accessibles ainsi à un plus grand nombre et dans des endroits reculés.

Cependant, la collectivisation des outils et des accès à Internet pose des problèmes organisationnels et institutionnels : qui gère les accès ? un individu ? une entreprise ? une coopérative ? une association ? Quels sont les mécanismes mis en place pour assurer la pérennité des centres, notamment la maintenance des équipements informatiques, la formation du personnel, les coûts de fonctionnement et de connexion?

Dans un environnement concurrentiel, les centres d'accès gérés par les entreprises privées peuvent proposer des avantages intéressants aux consommateurs. Cependant, ils ne sont souvent pas suffisants pour permettre aux populations à faible revenu d'accéder à ces services. Une association ou une coopérative - de gestion souvent plus délicate, compte tenu d'intérêts individuels parfois contradictoires au sein de la même structure - permet d'offrir des services plus adaptés aux populations défavorisées. Il leur faudra cependant mettre en place des procédures d'autogestion et d'autofinancement imaginatives pour pouvoir renouveler le stock de leur matériel et proposer des coûts permettant de couvrir les frais de fonctionnement.

Grâce aux recettes générées par ses formations et ses services de maintenance, Yam Pukri s'autofinance à $50 \%$. La multiplication des centres d'accès à Internet a réduit ses sources de revenu. Certains bénéficient même de lignes spécialisées gratuites dans le cadre de programmes de coopération et peuvent offrir des prix à la baisse. Dans la pratique, l'aspect coût n'est cependant pas le plus important. $\mathrm{Ce}$ qui est fondamental, c'est d'accompagner les utilisateurs par des conseils, de la formation et des informations sur le contexte plus global des TIC. 
Un nouveau créneau est par exemple d'aider les petites entreprises et les associations à gérer et maintenir leurs télécentres. Yam Pukri intervient aussi pour soutenir les associations recevant du matériel informatique du Nord à mieux l'utiliser.

Quant aux partenaires européens de l'association, ils lui permettent de créer des contenus locaux qui sont par définition non rentables. Après avoir défini ses priorités, Yam Pukri essaie d'être en phase avec ses partenaires européens, qui sont souvent intéressés par des résultats à court terme, ce qui rend les collaborations parfois difficiles. L'association peine à tenir les délais définis à l'avance, car d'autres composantes de la société ralentissent le rythme de réalisation des programmes. Par exemple, les grèves des étudiants perturbent les formations dans les établissements scolaires. Le développement des TIC est une course de fond, et les partenaires de la coopération internationale devraient davantage réfléchir à la pérennité de leurs actions qu'aux résultats spectaculaires à court terme.
La collaboration entre Yam Pukri et Terre des Hommes Genève ${ }^{2}$ est en cela exemplaire, car elle garantit à l'association un fond de roulement pour son fonctionnement. Elle permet aussi de développer des contenus et d'animer un forum pour les jeunes sur des sujets comme les droits de l'homme, la migration, l'intolérance ou le travail des enfants ${ }^{3}$. Grâce à la collaboration avec l'IICD ${ }^{4}$ (Hollande), un site dynamique $^{5}$ a été créé pour publier des actualités et des expériences locales en matière de TIC. Un autre site ${ }^{6}$ facilite la publication de contenus provenant d'associations burkinabés.

Au moment de la création de l'association, les accès collectifs à Internet au Burkina Faso se comptaient sur les doigts d'une main. Quelques années plus tard, ils se sont multipliés malgré la mauvaise connectivité du pays. Le public a donc un choix beaucoup plus large d'accès et de services, ce qui est un vrai progrès. Cependant, en ce début de millénaire, le Burkina Faso a atteint un seuil de développement qu'il ne pourra pas dépasser si les infrastructures ne sont pas développées, notamment dans les zones rurales.

\footnotetext{
$<$ www.tdh-geneve.ch $>$.

$<$ http://tecfaseed.unige.ch/tdh $>$.

International Institute for Communication and Development, $<w w w . i i c d . o r g>$.

$<w w w . b u r k i n a-n t i c . o r g>$.

$<$ www.faso-ong.org $>$.
} 